\title{
Mechanical Loading Improves Tendon- Bone Healing in a Rabbit Anterior Cruciate Ligament Reconstruction Model by Promoting Proliferation and Matrix Formation of Mesenchymal Stem Cells and Tendon Cells
}

\author{
Fanglong Song Dawei Jiang Tianchen Wang Yi Wang Fengmei Chen \\ Guoxing Xu Yinquan Zhang Yifan Kang \\ Department of Orthopedics, Third Affiliated Hospital of PLA Second Military Medical University, \\ Shanghai, China
}

\section{Key Words}

Mechanical loading - Tendon-bone healing • Mesenchymal stem cells • Tendon cells • Coculture

\footnotetext{
Abstract

Background/Aims: This study investigated the effect of mechanical stress on tendon-bone healing in a rabbit anterior cruciate ligament (ACL) reconstruction model as well as cell proliferation and matrix formation in co-culture of bone-marrow mesenchymal stem cells (BMSCs) and tendon cells (TCs). Methods: The effect of continuous passive motion (CPM) therapy on tendon-bone healing in a rabbit $A C L$ reconstruction model was evaluated by histological analysis, biomechanical testing and gene expressions at the tendon-bone interface. Furthermore, the effect of mechanical stretch on cell proliferation and matrix synthesis in BMSC/TC co-culture was also examined. Results: Postoperative CPM therapy significantly enhanced tendon-bone healing, as evidenced by increased amount of fibrocartilage, elevated ultimate load to failure levels, and up-regulated gene expressions of Collagen I, alkaline phosphatase, osteopontin, Tenascin $\mathrm{C}$ and tenomodulin at the tendon-bone junction. In addition, BMSC/TC co-culture treated with mechanical stretch showed a higher rate of cell proliferation and enhanced expressions of Collagen I, Collagen III, alkaline phosphatase, osteopontin, Tenascin C and tenomodulin than that of controls. Conclusion: These results demonstrated that proliferation and differentiation of local precursor cells could be enhanced by mechanical stimulation, which results in enhanced regenerative potential of BMSCs and TCs in tendon-bone healing. 


\section{Introduction}

The anterior cruciate ligament (ACL) plays an important role in stabilization of the knee joints and is one of the most commonly injured ligaments, particularly in young athletes [1]. In response to injury, the ACL has limited regenerative potential due to its limited blood supply and unique intra-articular environment [2-4]. Furthermore, ACL injury is often associated with an increased risk of early osteoarthritis of the knee [5, 6]. Several methods of ACL reconstruction using autografts or allografts of the hamstrings or patellar tendon have been under investigation in the last decades for the management of ACL tears $[1,7]$. However, the long-term outcomes of current reconstruction methods remain unsatisfactory with high failure rates, thus revisions are necessary [8].

In ACL reconstruction, passing the tendon graft through a bone tunnel is often required, which renders tendon-bone healing challenging, as this process requires the healing of two inhomogeneous tissues [7]. Previous studies have demonstrated that the structure of tendon-bone healing after ACL reconstruction differs dramatically from the native enthesis tissue connecting tendon to bone $[9,10]$. The poor quality of tendon-bone healing always leads to formation of scar tissue and inferior mechanical properties [11].

The distinct mechanical stress environment at the interface of the tendon-bone results from the unique tissue structure in this area, which involves two materials of widely different mechanical properties: tendons/ligaments and bone [12-14]. At the same time, mechanical stress is particularly critical for the formation of the tendon-bone juntion structure [9, 12 , 15]. As often occurs in bone fractures, micromovement around the fragments is beneficial to fracture healing, thus postoperative functional exercise after joint surgeries is often recommended to restore joint function $[16,17]$. Continuous passive motion (CPM) therapy, as an effective adjunctive treatment for osteochondral pathologies afflicting the knee, is widely used after joint replacement, fixation of intra-articular fractures, release of arthrofibrosis/adhesive capsulitis, and regenerative surgeries for microfractures [17]. Intensive preclinical animal studies and clinical studies have focused on the role of CPM therapy in ACL reconstruction, but the clinical effects of CPM therapy remain controversial [17-22]. In addition, although CPM therapy of the knee after ACL reconstruction offers a cyclic mechanical environment at the interface of the tendon-bone, there have been few reports on its effect on tendon-bone healing. It seems that the most accepted evaluation methods of the tendon-bone healing quality are histological and mechanical analysis, which can be readily performed in animal studies, but not in human studies. Another reason is that the parameters of CPM therapy, such as initiation time, intensity, frequency, and duration, vary between studies and, at present, there are no widely accepted criteria [17].

Mesenchymal stem cells (MSCs) are regarded as a key factor in response to tendonbone injury, as they pass through holes drilled at the tendon footprint through the bone marrow, infiltrate around the tendon graft, and participate in the healing [23]. Tendon grafts reportedly develop necrosis followed by revascularization and tendon cells (TCs) have limited potential for proliferation and differentiation, thus precursor cells, such as tendonderived MSCs (TDMSCs), are important for formation of the fibrocartilage junction in tendon-bone healing [7]. Since the two sources of MSCs in the tendon-bone interface possess efficient features for the reconstruction and remodeling during tendon-bone healing, recent intensive studies have focused on the use of MSCs to accelerate early tendon-bone healing and have shown promising results [24-27]. MSCs exposed to mechanical stimulation have been demonstrated to exert enhanced proliferation and differentiation, as well as inhibition of apoptosis [28-32]. Thus, mechanical loading may improve tendon-bone healing by promoting the regenerative potential of MSCs.

The hypothesis of the present study was that cyclic mechanical loading might enhance tendon-bone healing by promoting cell proliferation and matrix formation of precursor cells at the tendon-bone interface. To test this hypothesis, the contribution of CPM therapy to tendon-bone healing after ACL reconstruction surgery was evaluated in a rabbit model by histological and biomechanical evaluations and reverse transcription polymerase 
Song et al.: Mechanical Loading Improves Tendon-Bone Healing by Promoting Regenerative Potential of Cells

chain reaction (RT-PCR) analysis of gene expressions of Collagen I (COL1A1), Collagen II (COL2A1), Collagen III (COL3A1), alkaline phosphatase (ALP), osteopontin (OPN), Tenascin $\mathrm{C}$ and tenomodulin (TNMD) at the tendon-bone interface. Furthermore, the effects of mechanical stretch on a co-culture of bone marrow-derived MSCs (BMSCs) and TCs were also determined by detecting the proliferation and matrix synthesis of the two cell types.

\section{Materials and Methods}

\section{Study designing and parameter setting}

All the animal experiments were approved by the Animal Research Ethics Committee of PLA Second Military Medical University. A total of 72 male New Zealand white rabbits (6-month-old) weighing between 3-3.5kg were purchased from the PLA Second Military Medical University Animal Administration Center.

For animal experiment, all the experimental animals undergoing unilateral (right) knee ACL reconstruction with autologous semitendinosus tendon were randomly divided into two groups: control group $(n=36)$ and CPM-treated group $(n=36)$. ACL reconstructions in the control group were performed following by free activities in cages; animals in CPM-treated group underwent CPM of the right knees two weeks after the operation. Parameters of the CPM: $40^{\circ}-110^{\circ}$ of flexion; 1 cycle $/ 30 \mathrm{~s} \times 60 \mathrm{~min}$; every other day; 3 weeks. 18 specimens from each group were harvested at 6 and 12 weeks postoperatively and evaluated using histological observation $(n=6)$, biomechanical test $(n=6)$, and RT-PCR assay ( $n=6)$, respectively.

For in vitro experiment, bone marrow and semitendinosus tendon were harvested from rabbits for isolation and culture of BMSCs and TCs. Differentiation assay was performed to identify the multiple differentiation potential of BMSCs. Co-culture system of the two cells (1:1) seeded on collagen I-coated sixwell Bioflex plates was used to explore the effect of mechanical stretch on the proliferation and synthesis of extracellular matrix in the two sources of cells. Parameters of the mechanical stretch: magnitude: $10 \%$ elongation; duration: $48 \mathrm{~h}$; frequency: 10 cycles/min with each cycle consisting of $2 \mathrm{~s}$ of stretch and $2 \mathrm{~s}$ of relaxation. After mechanical stimulation, cell proliferation was determined and total RNA was isolated from the cultured cells and RT-PCR was performed.

\section{ACL reconstruction}

All animals were operated under general anesthesia with 3\% intraperitoneal sodium phenobarbital $(1.0 \mathrm{~mL} / \mathrm{kg})$. A middle anterior incision was made and the semitendinosus tendon $(2.5 \mathrm{~cm})$ was isolated and harvested from posteromedial part of the knee joint through a small incision (Fig. 1A). As weaving suture was prepared on each end of the tendon graft with a 2-0/T silk braided non-absorbable suture (Ethicon; Johnson \& Johnson, USA) (Fig. 1B), the cavity of knee joint was exposed through a medial parapatellar approach and the native ACL was fully resected with a sharp surgical blade. Then the bone tunnels were drilled through femur and tibia at the footprint of the native ACL using a $1.5 \mathrm{~mm}$ Kirschner wire. The tendon

Fig. 1. Surgical procedures for ACL reconstruction using autologous semitendinosus tendon. (A, B) The semitendinosus tendon was harvested and weaving sutures were prepared on each end of the tendon autograft. (C) Tendon graft insertion into tibial and femoral tunnels. (D) Straddle nails made with a needle of a $10 \mathrm{ml}$ syringe. (E, F) The tendon graft was fixed adjacent to the tunnel exit by knotting at straddle nails.

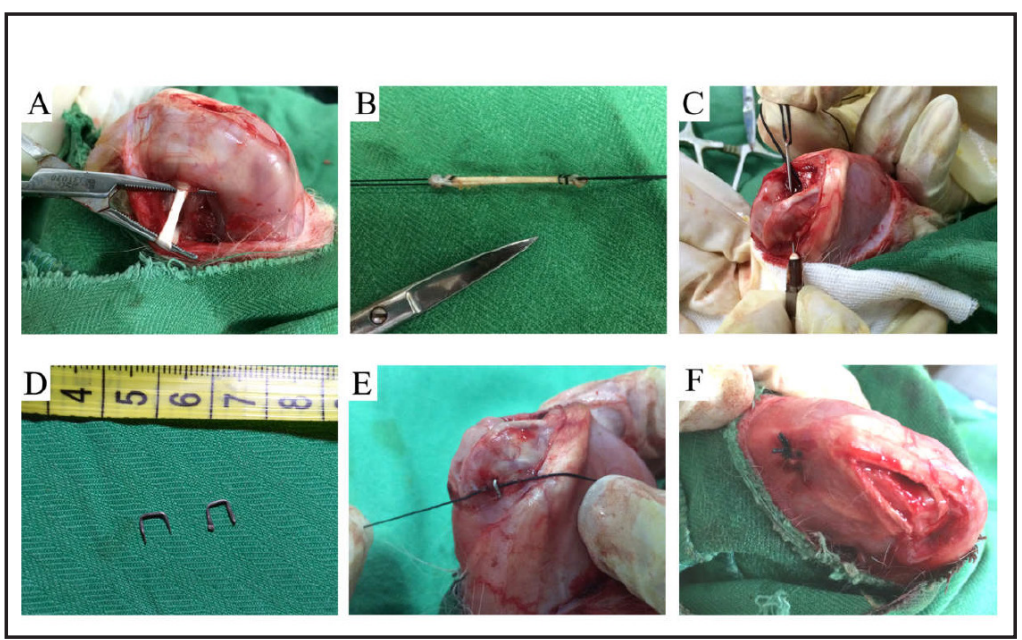




\section{Cellular Physiology Cell Physiol Biochem 2017;41:875-889

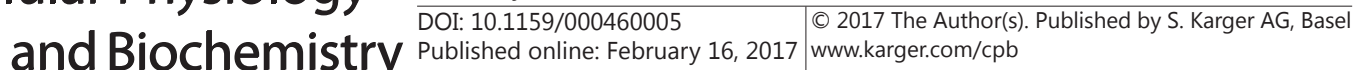

Song et al.: Mechanical Loading Improves Tendon-Bone Healing by Promoting Regenerative Potential of Cells

Table 1. Modified Histologic Scoring System for Tendon-Bone Healing

\begin{tabular}{|c|c|c|c|c|}
\hline Cellularity & $\begin{array}{l}1 \\
\text { Marked }\end{array}$ & $\begin{array}{l}2 \\
\text { Moderate } \\
\end{array}$ & $\begin{array}{l}3 \\
\text { Mild }\end{array}$ & $\begin{array}{l}4 \\
\text { Minimal }\end{array}$ \\
\hline Mature cartilage cells & $<25 \%$ & $25-50 \%$ & $50-75 \%$ & $>75 \%$ \\
\hline $\begin{array}{l}\text { Extent of fibrocartilage tissue } \\
\text { surrounding tendon }\end{array}$ & $\begin{array}{l}\text { Slightly } \\
\text { surrounded } \\
(<25 \%)\end{array}$ & $\begin{array}{l}\text { Partially surrounded } \\
{[25-50 \%]}\end{array}$ & $\begin{array}{l}\text { Moderately } \\
\text { surrounded (50-75\%) }\end{array}$ & $\begin{array}{l}\text { Mostly } \\
\text { surrounded } \\
(>75 \%)\end{array}$ \\
\hline $\begin{array}{l}\text { Interface tissue transition from } \\
\text { bone to tendon }\end{array}$ & $\begin{array}{l}\text { Slightly } \\
\text { indistinct } \\
(<25 \%)\end{array}$ & $\begin{array}{l}\text { Partially indistinct } \\
(25-50 \%)\end{array}$ & $\begin{array}{l}\text { Moderately indistinct } \\
(50-75 \%)\end{array}$ & $\begin{array}{l}\text { Mostly } \\
\text { indistinct } \\
(>75 \%)\end{array}$ \\
\hline
\end{tabular}

graft was then passed through both the bone tunnels using a bearded needle (Fig. 1C). The femoral side was fixed by knotting at a straddle nail made with a needle of a $10 \mathrm{ml}$ syringe fixed adjacent to the tunnel exit (Fig. 1D, E). The tibial side was fixed similar to the femoral side at a $30^{\circ}$ of flexion of the knee after pre-tensioning (Fig. 1F). The joint was irrigated with $0.9 \%$ saline solution followed by the capsule and skin closed with interrupted sutures. Postoperatively, all the animals received an intramuscular injection of $50000 \mathrm{U}$ of Gentamicin every 8 hours for the first 3 days following surgery to prevent infection.

Continuous passive motion (CPM)

Animals in CPM-treated group underwent CPM of the right knees two weeks after the operation. The apparatus and parameters of CPM used in our study are modified based on descriptions by Zarnett et al. [33]. Briefly, during the following three weeks, animals' knees were moved passively by the CPM apparatus for $60 \mathrm{~min}$ every other day in an awakened state. A range of motion from $40^{\circ}-110^{\circ}$ at a rate of 1 cycle every 30 s was adopted. At other times, animals in CPM-treated group together with the control animals were allowed for free activities in cages after surgery until they were sacrificed.

\section{Histological analysis}

Rabbits were sacrificed using air injection intravenously into ear margin at 6 and 12 weeks postsurgery. Rabbit knee joints were removed and fixed in $10 \%$ formalin solution followed by decalcification for 2 months. The tibial parts of the tissues were then embedded in paraffin, and serial $4 \mathrm{~mm}$ thick coronal sections across the middle of the tendon were made. For histologic examination, the tissue sections were stained with H\&E (hematoxylin and eosin) and toluidine blue. The stained sections were analyzed to determine the fibrocartilage formation at the tendon-bone interface which was comparable in locations in each group. The histological evaluations were performed independently using light microscopy by two observers in a blinded fashion. According to the criteria of the previous studies [34, 35], we developed a modified scoring system based on the subcategories of cellularity at the interface tissue, mature cartilage cells, extent of fibrocartilage tissue surrounding tendon and interface tissue transition from bone to tendon (Table 1). There were 6 specimens in each group at each time point for histological analysis. Representative histological images matching the conclusions were presented.

\section{Biomechanical analysis}

Rabbit knee joints were isolated and all periarticular soft tissues containing synovial membrane, collateral ligaments and capsule of joint that connecting the femoral and tibial part were removed except the grafted tendon. All scar tissues, sutures and internal fixations at the tibial tunnel exits were carefully removed. The femur-tendon graft-tibia complexes were mounted onto the mechanical testing machine allowing tensile loading along the axis of the graft (STA1224; Orientec, Tokyo, Japan). After preconditioning of the constructs, a load-to-failure test was performed at an elongation speed of $40 \mathrm{~mm} / \mathrm{min}$. If the site of graft failure was at femoral tunnel or mid-substance, the test would continue until the maximal pullout load of the graft in tibial tunnel was recorded (ultimate load to failure).

\section{Isolation and culture of cells}

BMSCs and TCs were isolated from 5-month-old male New Zealand white rabbits under general anesthesia. For BMSCs isolation and culture, an aliquot of bone marrow (10 ml) was aspirated from the proximal tibia end of donor rabbits with a $20 \mathrm{ml}$ heparinized sterile syringe. Then the aspirate was centrifuged at $1500 \mathrm{rpm}$ for $20 \mathrm{~min}$. Precipitated cells were suspended in general medium DMEM/F12 (Ham's F12: high glucose DMEM 1:1; Hyclone, Pittsburgh, USA) supplemented with 10\% fetal bovine serum (FBS; Hyclone, Pittsburgh, USA) and 1\% penicillin/streptomycin (P/S) (Hyclone, Pittsburgh, USA) (complete 


\section{Cellular Physiology Cell Physiol Biochem 2017;41:875-889 \\ \begin{tabular}{l|l|l} 
and Biochemistry Publisned onlIne: February 16, 2017 & $\begin{array}{l}\text { @ } 2017 \text { The Author(s). Published by S. Karger AG, Basel } \\
\text { www.karger.com/cpb }\end{array}$ \\
\hline
\end{tabular}}

Song et al.: Mechanical Loading Improves Tendon-Bone Healing by Promoting Regenerative Potential of Cells

culture medium), plated in a $25 \mathrm{~cm}^{2}$ plastic culture flask and cultured in a humidified atmosphere incubator containing $95 \%$ air and $5 \% \mathrm{CO}_{2}$ at $37^{\circ} \mathrm{C}$. After 7 days, red blood cells were washed with phosphate buffered saline (PBS) and fresh medium added. After 3 days, cells reached 90-95\% confluence and were passaged by trypsinization and recultured as passage one at a density of approximately $10^{4} \mathrm{cells} / \mathrm{cm}^{2}$. For TCs isolation and culture, a $10 \mathrm{~mm}$ long semitendinosus tendon from the same rabbit was harvested. The tendon sheath and surrounding paratenon were carefully removed, the tendon tissue was washed in sterile PBS and then aseptically minced into pieces about $1 \mathrm{~mm}^{3}$ and digested in $0.2 \%$ collagenase Type I (Sigma, St. Louis, MO, USA) for $60 \mathrm{~min}$ at $37^{\circ} \mathrm{C}$. Next, the resulting cell solution was filtered through a $70-\mathrm{mm}$ cell strainer (Becton Dickinson, Franklin Lakes, NJ) and centrifuged at 1500rpm for $5 \mathrm{~min}$. The isolated cells were washed in PBS and resuspended in complete culture medium and seeded at a density of $10^{4} \mathrm{cells} / \mathrm{cm}^{2}$ in a $25 \mathrm{~cm}^{2}$ plastic culture flask and cultured in a humidified atmosphere incubator at $37^{\circ} \mathrm{C}$ with $5 \% \mathrm{CO}_{2}$. After 5 days, the cells were washed with PBS to remove the non-adherent cells and fresh medium was added with medium changed every other day. Cells were passaged by trypsinization when they were $90-95 \%$ confluent and seeded at a density of $10^{4} \mathrm{cells} / \mathrm{cm}^{2}$ as passage one. All cells used in the present experiment were passage one.

\section{Differentiation assay}

BMSCs were subjected to osteogenic, adipogenic, and chondrogenic differentiation media to identify the multiple differentiation potential of BMSCs. Briefly, for adipogenic and osteogenic differentiation, BMSCs were incubated in six-well plates and expanding the cells to $100 \%$ confluency. Adipogenic differentiation was induced by medium containing DMEM/F12, 10\% FBS, $1 \mu \mathrm{M}$ dexamethasone, 0.5mM 3-isobutyl-1-

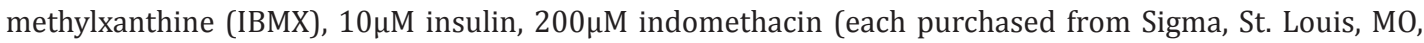
USA) and 1\% P/S. Medium was changed every 3 days. After 2 weeks, cells were fixed in 4\% paraformaldehyde and stained with Oil-red 0 solution. Osteogenesis was induced by medium containing DMEM/F12, 5\% FBS, 10nM dexamethasone, $10 \mathrm{mM} \beta$-glycerophosphate and $200 \mu \mathrm{M}$ L-ascorbic acid (each purchased from sigma, St. Louis, MO, USA) and 1\% P/S. Medium was changed every 3 days. After 2 weeks, cells were fixed in 4\% paraformaldehyde and stained with Alkalinephosphatase (ALP). Chondrogenesis was assessed by the pellet culture. A cell pellet was made by centrifugation of $1 \times 10^{6}$ cells at $1500 \mathrm{rpm}$ for $5 \mathrm{~min}$ and the pellet seeded onto PLGA $(8 \mathrm{~mm} \times 8 \mathrm{~mm} \times 2 \mathrm{~mm}$; polylactide (PLA): polyglycolic acid (PGA) 9:1; porosity 90\%; pore size $100 \mu \mathrm{m}$ ) and cultured with medium containing DMEM/F12, 5\% FBS, 10ng/mL TGF- $\beta$ (PeproTech, NJ, USA), 100nM dexamethasone, 10 $\mu \mathrm{M}$ insulin (all purchased from sigma, St. Louis, MO, USA) and 1\% P/S. The medium was changed every 2-3 days. After 3 weeks, the pellet was fixed in $4 \%$ paraformaldehyde and embedded in paraffin and sectioned. Representative sections were stained with toluidine blue to assess the glycosaminoglycan formation in the extracellular matrix of cells.

\section{Co-culture assay}

For direct-contact co-culture of BMSCs and TCs, the cells were seeded on collagen I-coated six-well Bioflex plates (Flexcell International, Hillsborough, NC, USA) with a density of $10^{4} \mathrm{cells} / \mathrm{cm}^{2}$ at the ratio of 1:1 (BMSCs/TCs, n=3). The complete culture medium was used for all co-culture experiments and was changed every 3 days.

\section{Mechanical stretch application}

For mechanical stretch, cells were seeded onto collagen I-coated six-well Bioflex plates at a density of $10^{4} \mathrm{cells} / \mathrm{cm}^{2}$. When cells were at $95 \%$ confluence after $48 \mathrm{~h}$, the medium was changed with DMEM/F12 for serum-starving for $24 \mathrm{~h}$ and then subjected to cyclic mechanical stretch using the Flexcell FX-5000T strain system (Flexcell International, NC, USA). Cells were stretched with a $10 \%$ elongation magnitude for $48 \mathrm{~h}$ at a frequency of 10 cycles/min, each cycle consisting of $2 \mathrm{~s}$ of stretch and $2 \mathrm{~s}$ of relaxation. Cells cultured on the same kind of plates without stretch loading were used as control cells.

\section{Cell proliferation assay}

Alamar Blue (AB, Alamar Biosciences, Sacramento, CA, USA) assay was performed to obtain the index of cell growth after stretch was applied to the mixed cells. In brief, after mechanical stimulation, fresh culture medium containing $10 \% \mathrm{AB}$ solution was added to cells and incubated at $37^{\circ} \mathrm{C}$ for $2 \mathrm{~h}$. When the $\mathrm{AB}$ mixture turned to a purplish or reddish shade, triplicates of $100 \mu \mathrm{L} \mathrm{AB}$ mixture from each well were transferred to 
Regenerative Potential of Cells

Table 2. Primer sequences for RT-PCR analysis

\begin{tabular}{llc}
\hline \multicolumn{1}{c}{ Gene } & \multicolumn{1}{c}{ Primer 5' to 3' $^{\prime}$ Fragment (bp) } \\
\hline COL1A1 & F:ACCTGGTCCCCAAGGTTTCCAA & 247 \\
XM_017348831.1 & R:CTTGGCACCATCCAAACCACTG & \\
COL2A1 & F:TGCAGGAGGGGAAGAGGTATAATG & 223 \\
NM_001195671.1 & R:GGTTCTCCTTTCTGCCCCTTTGGT & \\
COL3A1 & F:TGGTCTTCCTGGTGAAAACGGA & \multirow{2}{*}{196} \\
XM_002712333.3 & R:TTCACCCTTAGCACCAGGGGAT & \\
ALP & F:TGTGCTGTGCAGCCTTGGGTGAGT & 240 \\
XM_017346489.1 & R:TGTGCAGAGAGAGGGGCAGTCAGT & \\
OPN & F:GCAGCAACCACAGTTTTCACTG & 222 \\
D16544.1 & R:TACATTCAGGTGCTGAGCCACT & \\
Tenascin C & F:AGGGCTTTGAGGAAAGTGAACC & 216 \\
FJ480400.1 & R:TCAGAGCATACTCCACCGTGTT & \\
TNMD & F:GAACAAAATGAGCAGTGGGTGGTC & 222 \\
NM_001109818.1 & R:TTGCAAGGCATGATGACACGACAG & \\
$\beta$-actin & F:CAACTGGGACGACATGGAGAAG & \multirow{2}{*}{152} \\
NM_001101683.1 & R:TGAACGTCTCGAACATGATCTG & \\
\hline
\end{tabular}

a 96-well plate. Then, the absorbance was detected using an enzyme-linked immunosorbent assay reader (Molecular Devices, Sunnyvale, CA, USA) at 570 and $600 \mathrm{~nm}$. The number of viable cells was expressed as percentage of $\mathrm{AB}$ reduction (\% AB reduction) which was calculated according to the manufacturer's protocol.

\section{RT-PCR}

Total RNA was isolated from tendon-bone interfacial samples of the proximal tibia at the site in accordance with histologic examination or cultured cells using TRIzol reagent (Invitrogen, Carlsbad, CA, USA) according to the manufacturer's protocol. A spectrophotometer was used to determine RNA concentrations, and samples with values between 1.7 and 2.0 were used. Complementary DNA (cDNA) synthesis was carried out from RNA using a PrimeScript RT reagent kit (TaKaRa, Tokyo, Japan). Real-time PCR was performed using SYBR Premix Ex Taq (TaKaRa, Tokyo, Japan) with specific primers to determine the gene expression levels of different samples. The specificities of the PCR products were confirmed by conducting melting curve analysis, and the levels of mRNA were calculated as target gene expression normalized to $\beta$-actin. The primers used are shown in Table 2.

\section{Statistical analysis}

All data were expressed as mean \pm standard deviation (SD). A Student's t-test was used to evaluate results between two groups except for the evaluations of differences of gene expressions between groups, which a nonparametric Wilcoxon signed-rank test was performed. All the data analysis was performed with SPSS analysis software (SPSS Inc, 16.0). $p<0.05$ was regarded as statistically significant.

\section{Results}

\section{Clinical observations}

All animals recovered after surgery in 1-2h. There was no evidence of infection, immobilization or unloading of the joint in any of the animals during the experiments.

\section{Histological analysis of the tendon-bone healing after ACL reconstruction}

As shown in Fig. 2, 6 weeks after surgery, there was an obvious zone with highly cellular, disordered fibrovascular tissue at the tendon-bone interface in the control specimens and no evidence of cartilage tissue formation existed (Fig. 2Ba, e). In the CPM-treated specimens, healing occurred with the formation of new cartilage tissue between the tendon and bone. The tendon was moderately surrounded with chondrocytes with irregular cell shape (hypertrophic chondrocytes), however, the arrays of chondrocyte were along the direction of the force on tendon and the chondrocytes showed a vigorous extracellular matrix production. Fiber continuity occurred and the borderline between the tendon and bone was vague (Fig. 2Bb, f). 
12 weeks after surgery, occasional cartilage formed and the amount of cellular and vascular tissue decreased at the interface zone in the control specimens compared with that of 6 weeks. However, arrays of cells were irregular and less extracellular matrix of the chondrocytes synthesized compared with the CPM-treated specimens (Fig. 2Bc, g). 12 weeks after surgery, specimens in CPM-treated group demonstrated a typical direct insertion of ACL with progressive formation of new fibrocartilage and the cartilage tissue was more mature at the tendon-bone interface. Chondrocytes arranged in neat rows along the direction of the force on tendon, a vague tidal line formed between the fibrocartilage zone and calcified cartilage zone (Fig. 2Bd, h).

Of the two groups, histologic scores of tendon-bone healing increased significantly with time respectively $(p<0.05)$. At 6 and 12 weeks postoperatively, the CPM-treated group displayed a statistically significant higher histologic score, compared with that of control group respectively $(p<0.05)$, which indicated that CPM was a beneficial factor for the regeneration of tendon-bone junction (Fig. 3).

\section{Biomechanical testing of the joint after ACL reconstruction}

Biomechanical testing was performed 6 and 12 weeks after surgery (Fig. 4). The results showed that the average ultimate load to failure increased significantly in the two groups with time, respectively $(p<0.05)$. The pullout load of specimens in the CPM-treated group

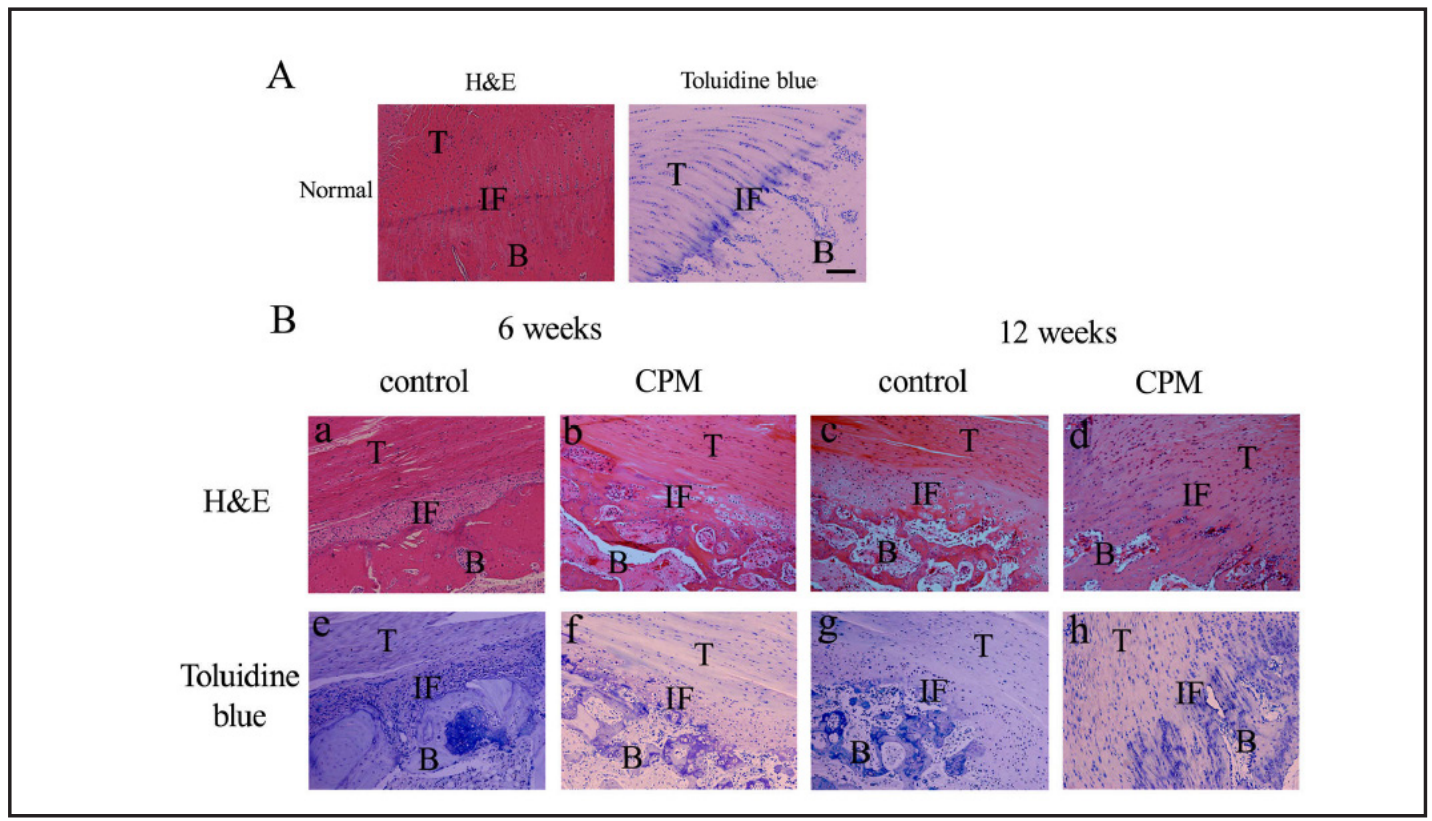

Fig. 2. Histological observations of tendon-bone healing. (A) Representative photographs of the direct insertion of normal ACL. Typical four layers of direct insertion of ACL were shown: tendon, fibrocartilage, calcified cartilage and bone and a clear tide line between the fibrocartilage zone and calcified cartilage zone was observed. (B) Representative histological photographs of specimens of control and CPM-treated groups 6 and 12 weeks postoperatively. (a, e) There was an obvious zone with highly cellular, disordered fibrovascular tissue at the tendon-bone interface and no evidence of cartilage tissue formation existed. (b, f) New cartilage tissue formed between the tendon and bone. The tendon was moderately surrounded with chondrocytes and the arrays of which were along the direction of the force on tendon. (c, g) Occasional cartilage formed and the amount of cellular and vascular tissue decreased at the interface zone and arrays of cells were irregular and less extracellular matrix of the chondrocytes synthesized. (d, h) Progressive formation of new fibrocartilage and the cartilage tissue was more mature at the tendon-bone interface. Chondrocytes arranged in neat rows along the direction of the force on tendon and a vague tidal line formed between the fibrocartilage zone and calcified cartilage zone. $\mathrm{N}=6$ from each group. Bars $=100 \mu \mathrm{m}$. T, tendon; $\mathrm{B}$, bone; IF, interface.

\section{KARGER}




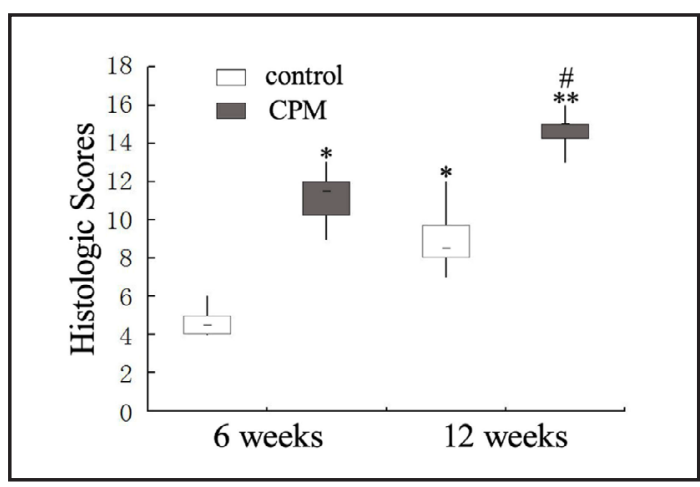

Fig. 3. Histologic scores of tendon-bone healing. Boxplots showing the histologic scores of control and CPM-treated groups at weeks 6 and 12 postoperatively. ${ }^{*} p<0.05$ versus control group at week $6 ;{ }^{* *} p$ $<0.05$ versus control group at week $12 ;{ }^{*} p<0.05$ versus CPM-treated group at week $6 . \mathrm{N}=6$ from each group.

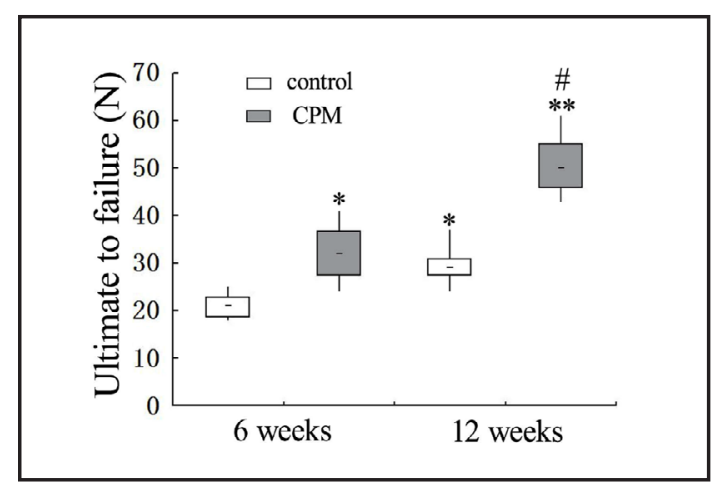

Fig. 4. Biomechanical testing of the joints after ACL reconstruction. Boxplots showing the ultimate load to failure of control and CPM-treated groups at weeks 6 and 12 postoperatively. ${ }^{*} p<0.05$ versus control group at week $6 ;{ }^{* *} p<0.05$ versus control group at week $12 ;{ }^{\#} p<0.05$ versus CPM-treated group at week $6 . \mathrm{N}=6$ from each group.

was significantly greater than that of control at 6 and 12 weeks after surgery, respectively $(p<0.05)$.

\section{Gene expressions at the interface between tendon and bone}

Relative mRNA expressions for bone, cartilage and ligament matrix formation at the interface between tendon and bone were evaluated using RT-PCR (Fig. 5). Expressions of COL1A1, ALP, OPN, Tenascin C and TNMD increased significantly with time respectively $(p<0.05)$ and their expressions in the CPM-treated group were significantly greater than that of control at different time after surgery, respectively ( $p<0.05$ ) (Fig. 5A, D-G). In the control group, gene expression of COL2A1 at week 12 was greater than that of week $6(p<0.05)$. However, COL2A1 expression decreased with time from week 6 to week 12 in the CPMtreated group $(p<0.05)$. Furthermore, CPM led to a significant up-regulation of COL2A1 at week 6 and down-regulation of it at week 12, compared with that of control $(p<0.05)$ (Fig. 5B).

Expression of COL3A1 in the two groups decreased significantly with time respectively $(p<0.05)$ and CPM induced a significant down-regulation of it $(p<0.05)$ at each time point respectively which showed an opposite tendency with COL1A1 (Fig. 5C).

\section{Cell observation and identification}

As shown in Fig. 6, BMSCs and TCs in the first passage were almost fibroblast-like cells with adherent growth ability (Fig. 6A, B). Oil-red staining of the BMSCs showed that moderate intracellular lipid droplets formed after adipogenic induction for 2 weeks (Fig. 6C). ALP staining after induction of osteogenesis for 2 weeks demonstrated the osteogenic potential of BMSCs (Fig. 6D). BMSCs pellet cultured in chondrogenic differentiation medium for 3 weeks were stained with toluidine blue, which indicated the secretion of proteoglycan (Fig. 6E). Results above suggested the multipotency of the BMSCs.

\section{Effect of mechanical stimulation on cell proliferation and gene expressions}

Co-culture of BMSCs with TCs treated with $10 \%$ mechanical strain for $48 \mathrm{~h}$ showed a higher rate of cell proliferation than control cells $(p<0.05)$ (Fig. 7). Additionally, mechanical strain influenced the expressions of the matrix-forming genes significantly (Fig. 8). Gene expressions of COL1A1, COL3A1, ALP, OPN, Tenascin C and TNMD in the Co-culture system were significantly up-regulated after stretching respectively $(p<0.05)$, however, expression of COL2A1 was not influenced by mechanical stretch (Fig. 8). 
Fig. 5. Gene expressions at the interface between tendon and bone. Relative mRNA expressions for (A) COL1A1, (B) COL2A1, (C) COL3A1, (D) ALP, (E) OPN, (F) Tenascin $C$ and (G) TNMD at the interface of tendon-bone in each group were analyzed at weeks 6 and 12 postoperatively. Levels of mRNA were normalized to $\beta$-actin and then related to the control (labeled as "1.00"). " $p<0.05$ versus control group at week $6 ;{ }^{* *} p<0.05$ versus control group at week $12 ;{ }^{\#} p<0.05$ versus CPM-treated group at week $6 . \mathrm{N}=6$ from each group.

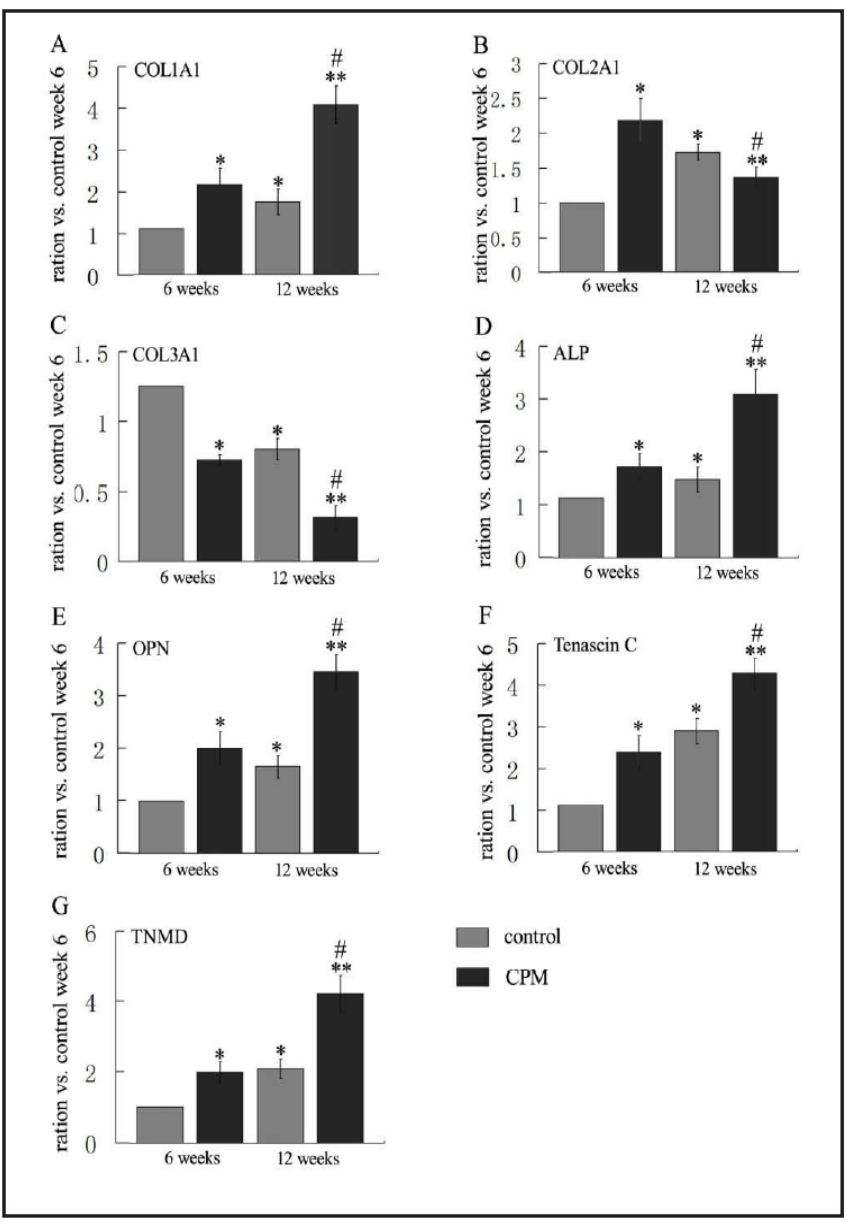

\section{Discussion}

In the present study, the efficacy of CPM therapy of the knee after ACL reconstruction surgery was investigated. Ideal ACL reconstruction should provide firm attachment of the tendon graft to the bone and minimize tendon-bone displacement under cyclic mechanical loading. The high initial stability of the tendon-bone junction allows earlier rehabilitation and quicker return to full activity [36]. Although the attachments of the two ends of the tendon graft are firm, the long distance between the biomechanical point of action of the ACL and the insertion site may result in relative movement of the tendon-bone junction during a large range of motion of the knee joint, which leads to expansion of the bone tunnel and loosening of fixations [37]. Additionally, it has been suggested that a delayed mechanical stimulation would provide an improved tendon-bone healing response compared with immediate initiation of mechanical loading or prolonged immobilization after ACL reconstruction [38]. Patients who had undergone early, aggressive rehabilitation after ACL reconstruction had been reported significantly greater tunnel widening compared with those who had undergone a conservative rehabilitation program [39]. Considering that early aggressive postoperative rehabilitation may have detrimental effects on graft-to-bone healing and any benefit of CPM therapy must be weighed against the risk of graft-tunnel motion, the intervention of therapy in this study was started 2 weeks after surgery to avoid blocking the infiltration of inflammatory cells and in-growth of vascular tissues, as well as to maintain the initial stability of the tendon graft.

Functional exercise is often recommended in clinical practice to accelerate rehabilitation after ACL reconstruction [16]. CPM therapy, although a classical choice for rehabilitation of knee injury, shows controversial effectiveness in returning knee function after ACL reconstruction [17-22, 40]. However, the effect of CPM therapy on the quality of tendon-bone 


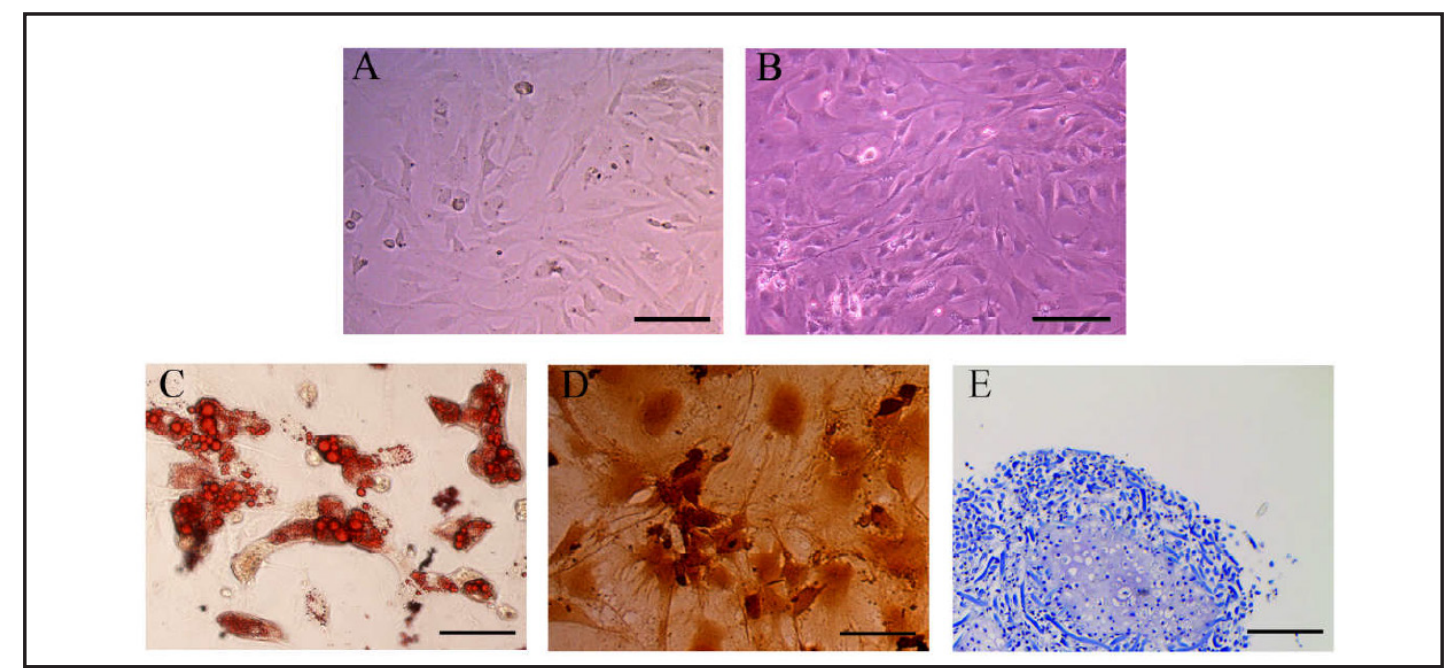

Fig. 6. Observation of BMSCs and TCs and identification of BMSCs by multi-lineage differentiation. (A, B) BMSCs and TCs at the first passage were almost fibroblast-like cells with adherent growth ability. Bars $=50 \mu \mathrm{m}$.

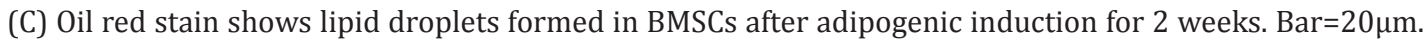

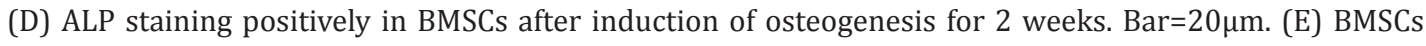
pellet cultured in chondrogenic differentiation medium for 3 weeks were stained positively with toluidine blue. Bar $=200 \mu \mathrm{m}$.

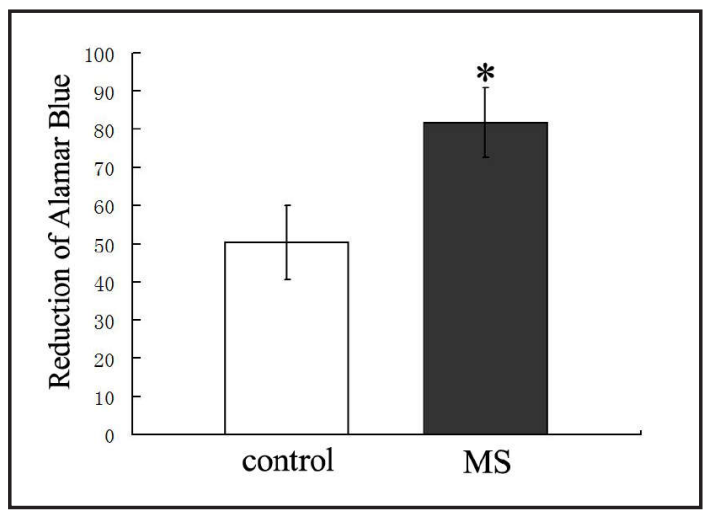

Fig. 7. Effect of mechanical stretch on cell proliferation of co-culture system of BMSCs and TCs. Data are presented as the mean \pm standard deviation of three independent experiments in triplicate. MS, mechanical stretch. ${ }^{*} p<0.05$ versus control group.

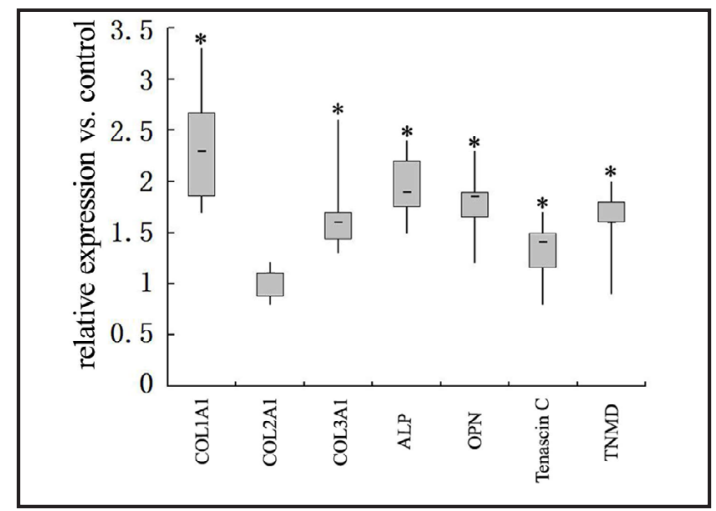

Fig. 8. Effect of mechanical stretch on gene expressions in co-culture system of BMSCs and TCs. Levels of mRNA were normalized to $\beta$-actin and then related to the control (labeled as "1.00"). Boxplots showing 6 independent experiments performed with different donors. ${ }^{*} p<0.05$ versus control group.

healing has been rarely reported. As it is impossible to conduct quantitative and standard active functional exercise studies using rabbits, CPM therapy, therefore, seems to be an optimal method to investigate the effect of mechanical stress on tendon-bone healing. The standard criteria of CPM therapy for animals after ACL reconstruction at present is limited and there is high variability in the composition and time ranges of CPM protocols, which may lead to confusing results. Therefore, the parameters of CPM therapy were set as mentioned in the materials and methods section based on previous reports, although most of those studies focused on outcomes of injuries to the articular cartilage rather than the ACL $[17,33]$. The results of this study showed that appropriate CPM therapy enhanced early tendon-bone healing and promoted the formation of fibrocartilage tissue of the tendon-bone junction.

Significant differences in the tendon-bone healing process are evident in different regions of the bone tunnel, resulting from variable biological and mechanical environments 
Song et al.: Mechanical Loading Improves Tendon-Bone Healing by Promoting Regenerative Potential of Cells

[36]. At the interface of the tendon-bone, compressive stress is thought to occur on the lower side and tensile stress on the upper side (inner hole of the bone tunnel), while shear force is dominant inside the tunnel [15]. Results of histological analysis have revealed that compressive stress enhances cartilage formation and stretch promotes the process of tendonbone healing, while shear force has little effect on tendon-bone junction regeneration [10, 15]. Stasiak et al. once designed a novel knee joint fixation/distraction system to generate a cyclic axial loading on the tendon-bone interface in a rat model of ACL reconstruction [41]. In the present study, to obtain consistent variables, biomechanical evaluations of the tendon-bone healing were all performed at the tendon graft and tibia, and tissues for histological examination and PCR analysis were obtained at the interface of the tendon graft and proximal lateral tibia, where mechanical stretch is dominant. It has been suggested that mechanical stretch enhances the process of tendon-bone healing and promotes proliferation and differentiation of MSCs $[15,42]$. As the two most important cell sources at the tendonbone interface [43], BMSCs and TCs participate in the process of tendon-bone healing in unique biomechanical environments. Therefore, in accordance with the stress environment of the tendon-bone junction, the effect of mechanical stretch on MSCs and TCs was explored.

In the present study, the effects of mechanical loading on each of the two sources of cells were not investigated. A 1:1 co-culture of BMSCs and ACL cells was reported to have the highest ratio of COL1A1 and Tenascin C expression, as compared with other ratios [43]. Therefore, a 1:1 co-culture system of BMSCs and TCs was used to investigate mechanical stimulation on cells. Although the co-culture system offers as a specific biological environment for the two cell types, in which interactions could also lead to enhanced proliferation and differentiation of either type [43], the results of this study demonstrated that mechanical stimulation promoted proliferation and synthesis of tendon- (COL1A1, Tenascin C and TNMD) and bone- (COL1A1, ALP and OPN) related genes of the co-culture system. As has been verified by many studies, mechanical stretch up-regulates tendon-related genes in TCs and bone-related genes in BMSCs. Zhang et al. reported that mechanical stretch (4\%) promoted the expression of tendon-related genes, including COL1A1 and TNMD, in TCs and high mechanical stretching (8\%) increased the expression of both tendon-related (COL1A1 and TNMD) and non-tendon-related genes, such as lipoprotein lipase (adipocytes), Sox 9 (chondrocytes) and Runx2 (osteocytes) in TDMSCs [44]. In ACL cells, mechanical loading induced up-regulation of the tenocyte-relevant genes Tenascin C, fibronectin, COL1A1 and COL3A1. However, no stretch-induced effects on the expression of tendon-related genes were detected in BMSCs [45]. Intensive studies have demonstrated that physical stimuli can induce osteogenic lineage commitment of stem cells $[28,46]$. As the cells used in this study were a co-culture of BMSCs and TCs, the results reflected the effects of mechanical stimulation on these two different cell types. Therefore, both the tendon- and bone-related genes were upregulated as an effect of mechanical stretch in the co-culture system. However, in both types of MSCs, expression of COL2A1, which represents the chondrogenesis potential in BMSCs, was unchanged by stretch stimulation, which showed a different trend from the research of Zhang et al. which used TDMSCs [44]. This discrepancy may due to the following three reasons: (1) BMSCs have little spontaneous chondrogenic potential when cultured in general medium, especially when cultured in monolayers instead of three-dimensionally [47]; (2) Mechanical stress, especially compression, has a strong effect on chondrogenesis of BMSCs, which stretch stimulation may not $[48,49]$; (3) Differentiation of BMSCs may be affected by the biological environment of the co-culture system, which contains a large amount of TCs. As a consequence, tenogenic potential was enhanced instead of chondrogenesis, as confirmed by the results of this study.

When compared with cellular experiments, gene expression profiles of tissues at the tendon-bone interface showed an interesting phenomenon. First, gene expression of COL2A1 in the control group increased with time. However, in response to mechanical loading, COL2A1 expression was decreased from week 6 to week 12, significantly enhanced at week 6 , and then downregulated at week 12 , when compared with controls. It has been reported that cell proliferation occurs in the early phase of tendon-bone healing and COL2A1

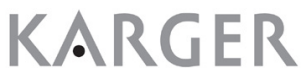


Song et al:: Mechanical Loading Improves Tendon-Bone Healing by Promoting Regenerative Potential of Cells

expression is up-regulated, then synthesis of COL2A1 decreases as the fibrocartilage tissue become mature [50]. The results of the present study suggest that mechanical stimulation may enhance the healing process and promote the mature phase. In addition, the differences of COL2A1 expression between cell and animal experiments may result from the more complex microenvironment, including expression of cytokines in different phases of the healing process, changes of the distributions and magnitudes of mechanical stress, and a greater variety of precursor cell lineages such as synovial-derived stem cells in vivo.

Second, COL3A1 expression decreased with time in the control group and was significantly down-regulated in vivo by mechanical loading, which differed from the results of the cellular experiment. It is reported that COL3A1 expression increased at week 6 and decreased at week 12 in a dog model of ACL reconstruction [51], which is in accordance with the results of the present study. During tendon healing, TCs produce large amounts of COL3A1 instead of COL1A1, which often results in scar formation [52]. In this study, significant regeneration accompanied by fibrocartilage formation, rather than scar formation, at the interface of tendon-bone was observed in the CPM-treated group, which suggested a more mature healing process. These results imply that although mechanical loading upregulated expression of COL3A1 in the co-culture system in vitro, the unique regenerative microenvironment in vivo may play a more important role than mechanical loading alone in the regulation of COL3A1 expression.

Although there were discrepancies in COL2A1 and COL3A1 gene expression levels, consistencies in expression profiles of tendon- and bone-related genes between the cellular experiment and animal study indicated that mechanical stimulation improved tendon-bone healing, at least partially, by promoting the regenerative potential of MSCs and TCs.

It has been suggested that regulation of the biological environment at the tendon-bone interface is crucial to improve regeneration of native tissues and to prevent scar formation [53]. The unique biological environment at the tendon-bone junction mostly promotes the natural course of endogenous grow factors $[54,55]$. Since it is difficult to mimic the kinetics of release of these factors at different stages of regeneration, MSCs isolated from bone marrow as well as many other tissues, such as adipose tissue [56], umbilical cord [34] and tendon tissues [7] have continued to attract attention in recent decades. MSCs have been reported to provide tendon-bone healing with an ideal bio-environment and accelerate tendon reintegration into bone to re-establish biomechanical properties. Other studies have shown that the high potential of MSCs to promote tendon-bone healing may result from: (1) the spontaneous migration of MSCs to an injured site due to the local inflammation environment [57]; (2) the biological and mechanical environment of the tendon-bone junction, which enhances proliferation and differentiation of MSCs into multiple lineages to repair damaged tissues [58, 59]; and (3) the "trophic" effects of MSCs by the secretion of different cytokines and molecules that promote angiogenesis, modulation of the inflammatory response, prevention of scar formation and recruitment of tendon intrinsic progenitor cells to the interface [60]. The results of this study demonstrated that proliferation and differentiation of local precursor cells could be enhanced by mechanical stimulation, which results in enhanced regenerative potential of MSCs and TCs in tendon-bone healing.

The several limitations to this study included (1) a relatively short observation time; (2) the need to further define the parameters of CPM therapy; (3) the failure to investigate the effects of mechanical loading on each of the two cell sources; (4) the lack of studies on the mechanisms of mechanical loading underlying the cell proliferation and matrix formation of the co-culture system; (5) since mechanical stimulation did not promote chondrogenic differentiation of the co-culture system, the formation of fibrocartilage at the tendon-bone interface is not well explained.

Altogether, the results of the present study attaches importance to mechanical loading in tendon-bone healing, as well as proliferation, differentiation and matrix formation of cells at the tendon-bone interface. Moreover, this study provides additional evidence to support the viewpoint that early rehabilitation exercise intervention promotes tendon-bone healing after ACL reconstruction. 


\section{Cellular Physiology Cell Physiol Biochem 2017;41:875-889 \\ \begin{tabular}{ll|l} 
DOI: 10.1159/000460005 & $\begin{array}{l}\text { O } 2017 \text { The Author(s). Published by S. Karger AG, Basel } \\
\text { www.karger.com/cpb }\end{array}$ \\
\cline { 2 - 4 }
\end{tabular} \\ Song et al.: Mechanical Loading Improves Tendon-Bone Healing by Promoting Regenerative Potential of Cells}

\section{Acknowledgments}

This work was supported by the National Natural Science Foundation of China (No.81371974).

\section{Disclosure Statement}

The authors declare that they have no competing interests.

\section{References}

-1 Hirzinger C, Tauber M, Korntner S, Quirchmayr M, Bauer HC, Traweger A, Tempfer H: ACL injuries and stem cell therapy. Arch Orthop Trauma Surg 2014;134:1573-1578.

-2 Amiel D, Nagineni CN, Choi SH, Lee J: Intrinsic properties of ACL and MCL cells and their responses to growth factors. Med Sci Sports Exerc 1995;27:844-851.

-3 Bray RC, Leonard CA, Salo PT: Vascular physiology and long-term healing of partial ligament tears. J Orthop Res 2002;20:984-989.

4 Murray MM, Martin SD, Martin TL, Spector M: Histological changes in the human anterior cruciate ligament after rupture. J Bone Joint Surg Am 2000;82:1387-1397.

5 Hill CL, Seo GS, Gale D, Totterman S, Gale ME, Felson DT: Cruciate ligament integrity in osteoarthritis of the knee. Arthritis Rheum 2005;52:794-799.

6 Nau T, Teuschl A: Regeneration of the anterior cruciate ligament: Current strategies in tissue engineering. World J Orthop 2015;6:127-136.

7 Lui PP, Wong OT: Tendon stem cells: experimental and clinical perspectives in tendon and tendon-bone junction repair. Muscles Ligaments Tendons J 2012;2:163-168.

-8 Yabroudi MA, Bjornsson H, Lynch AD, Muller B, Samuelsson K, Tarabichi M, Karlsson J, Fu FH, Harner CD, Irrgang JJ: Predictors of Revision Surgery After Primary Anterior Cruciate Ligament Reconstruction. Orthop J Sports Med 2016;4:2325967116666039.

-9 Carbone A, Carballo C, Ma R, Wang H, Deng X, Dahia C, Rodeo S: Indian hedgehog signaling and the role of graft tension in tendon-to-bone healing: Evaluation in a rat ACL reconstruction model. J Orthop Res 2016;34:641-649.

10 Rodeo SA, Arnoczky SP, Torzilli PA, Hidaka C, Warren RF: Tendon-healing in a bone tunnel. A biomechanical and histological study in the dog. J Bone Joint Surg Am 1993;75:1795-1803.

11 Chan BP, Fu SC, Qin L, Rolf C, Chan KM: Pyridinoline in relation to ultimate stress of the patellar tendon during healing: an animal study. J Orthop Res 1998;16:597-603.

12 Font Tellado S, Balmayor ER, Van Griensven M: Strategies to engineer tendon/ligament-to-bone interface: Biomaterials, cells and growth factors. Adv Drug Deliv Rev 2015;94:126-140.

13 Yang PJ, Temenoff JS: Engineering orthopedic tissue interfaces. Tissue Eng Part B Rev 2009;15:127-141.

14 Shaw HM, Benjamin M: Structure-function relationships of entheses in relation to mechanical load and exercise. Scand J Med Sci Sports 2007;17:303-315.

15 Yamakado K, Kitaoka K, Yamada H, Hashiba K, Nakamura R, Tomita K: The influence of mechanical stress on graft healing in a bone tunnel. Arthroscopy 2002;18:82-90.

-16 Nyland J, Mattocks A, Kibbe S, Kalloub A, Greene JW, Caborn DN: Anterior cruciate ligament reconstruction, rehabilitation, and return to play: 2015 update. Open Access J Sports Med 2016;7:21-32.

17 Knapik DM, Harris JD, Pangrazzi G, Griesser MJ, Siston RA, Agarwal S, Flanigan DC: The basic science of continuous passive motion in promoting knee health: a systematic review of studies in a rabbit model. Arthroscopy 2013;29:1722-1731.

18 Wright RW, Haas AK, Anderson J, Calabrese G, Cavanaugh J, Hewett TE, Lorring D, McKenzie C, Preston E, Williams G, Group M: Anterior Cruciate Ligament Reconstruction Rehabilitation: MOON Guidelines. Sports Health 2015;7:239-243.

19 Richmond JC, Gladstone J, MacGillivray J: Continuous passive motion after arthroscopically assisted anterior cruciate ligament reconstruction: comparison of short-versus long-term use. Arthroscopy $1991 ; 7: 39-44$ 


\section{Cellular Physiology Cell Physiol Biochem 2017;41:875-889 \begin{tabular}{l|l|l} 
DOI: 10.1159/000460005 & O 2017 The Author(s). Published by S. Karger AG, Basel
\end{tabular} and Biochemistry

Song et al.: Mechanical Loading Improves Tendon-Bone Healing by Promoting Regenerative Potential of Cells

20 Wright RW, Preston E, Fleming BC, Amendola A, Andrish JT, Bergfeld JA, Dunn WR, Kaeding C, Kuhn JE, Marx RG, McCarty EC, Parker RC, Spindler KP, Wolcott M, Wolf BR, Williams GN: A systematic review of anterior cruciate ligament reconstruction rehabilitation: part I: continuous passive motion, early weight bearing, postoperative bracing, and home-based rehabilitation. J Knee Surg 2008;21:217-224.

21 Bible JE, Simpson AK, Biswas D, Pelker RR, Grauer JN: Actual Knee Motion during Continuous Passive Motion Protocols is Less Than Expected. Clin Orthop Relat Res 2009;467:2656-2661.

22 Dorr LD: Continuous passive motion offers no benefit to the patient. Orthopedics 1999;22:393.

23 Kida Y, Morihara T, Matsuda K, Kajikawa Y, Tachiiri H, Iwata Y, Sawamura K, Yoshida A, Oshima Y, Ikeda T, Fujiwara H, Kawata M, Kubo T: Bone marrow-derived cells from the footprint infiltrate into the repaired rotator cuff. J Shoulder Elbow Surg 2013;22:197-205.

-24 Lim JK, Hui J, Li L, Thambyah A, Goh J, Lee EH: Enhancement of tendon graft osteointegration using mesenchymal stem cells in a rabbit model of anterior cruciate ligament reconstruction. Arthroscopy 2004;20:899-910.

-25 Soon MY, Hassan A, Hui JH, Goh JC, Lee EH: An analysis of soft tissue allograft anterior cruciate ligament reconstruction in a rabbit model - A short-term study of the use of mesenchymal stem cells to enhance tendon osteointegration. Am J Sports Med 2007;35:962-971.

26 Rizzello G, Longo UG, Petrillo S, Lamberti A, Khan WS, Maffulli N, Denaro V: Growth factors and stem cells for the management of anterior cruciate ligament tears. Open Orthop J 2012;6:525-530.

27 Gao Y, Zhang Y, Lu Y, Wang Y, Kou X, Lou Y, Kang Y: TOB1 Deficiency Enhances the Effect of Bone MarrowDerived Mesenchymal Stem Cells on Tendon-Bone Healing in a Rat Rotator Cuff Repair Model. Cell Physiol Biochem 2016;38:319-329.

-28 Chen JC, Jacobs CR: Mechanically induced osteogenic lineage commitment of stem cells. Stem Cell Res Ther 2013;4:107.

-29 Kelly DJ, Jacobs CR: The role of mechanical signals in regulating chondrogenesis and osteogenesis of mesenchymal stem cells. Birth Defects Res C Embryo Today 2010;90:75-85.

-30 Bin G, Bo Z, Jing W, Jin J, Xiaoyi T, Cong C, Liping A, Jinglin M, Cuifang W, Yonggang C, Yayi X: Fluid shear stress suppresses TNF-alpha-induced apoptosis in MC3T3-E1 cells: Involvement of ERK5-AKT-FoxO3aBim/FasL signaling pathways. Exp Cell Res 2016;343:208-217.

- 31 Aisha MD, Nor-Ashikin MN, Sharaniza AB, Nawawi H, Froemming GR: Orbital fluid shear stress promotes osteoblast metabolism, proliferation and alkaline phosphates activity in vitro. Exp Cell Res 2015;337:8793.

-32 Song F, Wang Y, Jiang D, Wang T, Zhang Y, Ma H, Kang Y: Cyclic Compressive Stress Regulates Apoptosis in Rat Osteoblasts: Involvement of PI3K/Akt and JNK MAPK Signaling Pathways. PLoS One 2016;11:e0165845.

33 Zarnett R, Velazquez R, Salter RB: The effect of continuous passive motion on knee ligament reconstruction with carbon fibre. An experimental investigation. J Bone Joint Surg Br 1991;73:47-52.

-34 Jang KM, Lim HC, Jung WY, Moon SW, Wang JH: Efficacy and Safety of Human Umbilical Cord Blood-Derived Mesenchymal Stem Cells in Anterior Cruciate Ligament Reconstruction of a Rabbit Model: New Strategy to Enhance Tendon Graft Healing. Arthroscopy 2015;31:1530-1539.

-35 Ide J, Kikukawa K, Hirose J, Iyama K, Sakamoto H, Fujimoto T, Mizuta H: The effect of a local application of fibroblast growth factor- 2 on tendon-to-bone remodeling in rats with acute injury and repair of the supraspinatus tendon. J Shoulder Elbow Surg 2009;18:391-398.

-36 Chen CH: Graft healing in anterior cruciate ligament reconstruction. Sports Med Arthrosc Rehabil Ther Technol 2009;1:21.

-37 Rodeo SA, Kawamura S, Kim HJ, Dynybil C, Ying L: Tendon healing in a bone tunnel differs at the tunnel entrance versus the tunnel exit: an effect of graft-tunnel motion? Am J Sports Med 2006;34:1790-1800.

-38 Bedi A, Kovacevic D, Fox AJ, Imhauser CW, Stasiak M, Packer J, Brophy RH, Deng XH, Rodeo SA: Effect of early and delayed mechanical loading on tendon-to-bone healing after anterior cruciate ligament reconstruction. J Bone Joint Surg Am 2010;92:2387-2401.

-39 Yu JK, Paessler HH: Relationship between tunnel widening and different rehabilitation procedures after anterior cruciate ligament reconstruction with quadrupled hamstring tendons. Chin Med J Engl 2005;118:320-326.

40 Gatewood CT, Tran AA, Dragoo JL: The efficacy of post-operative devices following knee arthroscopic surgery: a systematic review. Knee Surg Sports Traumatol Arthrosc 2016;10.1007/s00167-016-4326-4. 


\section{Cellular Physiology Cell Physiol Biochem 2017;41:875-889 \begin{tabular}{c|c|c|c|} 
DOI: 10.1159/000460005 & O 2017 The Author(s). Published by S. Karger AG, Basel
\end{tabular} and Biochemistry

-41 Stasiak M, Imhauser C, Packer J, Bedi A, Brophy R, Kovacevic D, Jackson K, Deng XH, Rodeo S, Torzilli P: A Novel In Vivo Joint Loading System to Investigate the Effect of Daily Mechanical Load on a Healing Anterior Cruciate Ligament Reconstruction. J Med Device 2010;4:15003.

-42 Maul TM, Chew DW, Nieponice A, Vorp DA: Mechanical stimuli differentially control stem cell behavior: morphology, proliferation, and differentiation. Biomech Model Mechanobiol 2011;10:939-953.

43 Canseco JA, Kojima K, Penvose AR, Ross JD, Obokata H, Gomoll AH, Vacanti CA: Effect on ligament marker expression by direct-contact co-culture of mesenchymal stem cells and anterior cruciate ligament cells. Tissue Eng Part A 2012;18:2549-2558.

-44 Zhang J, Wang JH: The Effects of Mechanical Loading on Tendons-An In Vivo and In Vitro Model Study. PLoS One 2013;8:e71740.

45 Kreja L, Liedert A, Schlenker H, Brenner RE, Fiedler J, Friemert B, Durselen L, Ignatius A: Effects of mechanical strain on human mesenchymal stem cells and ligament fibroblasts in a textured poly(L-lactide) scaffold for ligament tissue engineering. J Mater Sci Mater Med 2012;23:2575-2582.

46 Liu X, Chen W, Zhou Y, Tang K, Zhang J: Mechanical Tension Promotes the Osteogenic Differentiation of Rat Tendon-derived Stem Cells Through the Wnt5a/Wnt5b/JNK Signaling Pathway. Cell Physiol Biochem 2015;36:517-530.

47 Jung Y, Kim SH, Kim YH, Kim SH: The effects of dynamic and three-dimensional environments on chondrogenic differentiation of bone marrow stromal cells. Biomed Mater 2009;4:055009.

- 48 Pelaez D, Arita N, Cheung HS: Extracellular signal-regulated kinase (ERK) dictates osteogenic and/or chondrogenic lineage commitment of mesenchymal stem cells under dynamic compression. Biochem Biophys Res Commun 2012;417:1286-1291.

49 Haudenschild AK, Hsieh AH, Kapila S, Lotz JC: Pressure and distortion regulate human mesenchymal stem cell gene expression. Ann Biomed Eng 2009;37:492-502.

50 Gimbel JA, Van Kleunen JP, Lake SP, Williams GR, Soslowsky LJ: The role of repair tension on tendon to bone healing in an animal model of chronic rotator cuff tears. J Biomech 2007;40:561-568.

51 Xie X, Wu H, Zhao S, Xie G, Huangfu X, Zhao J: The effect of platelet-rich plasma on patterns of gene expression in a dog model of anterior cruciate ligament reconstruction. J Surg Res 2013;180:80-88.

-52 Liu CF, Aschbacher-Smith L, Barthelery NJ, Dyment N, Butler D, Wylie C: What we should know before using tissue engineering techniques to repair injured tendons: a developmental biology perspective. Tissue Eng Part B Rev 2011;17:165-176.

53 Lorbach O, Baums MH, Kostuj T, Pauly S, Scheibel M, Carr A, Zargar N, Saccomanno MF, Milano G: Advances in biology and mechanics of rotator cuff repair. Knee Surg Sports Traumatol Arthrosc 2015;23:530-541.

-54 Kobayashi M, Itoi E, Minagawa H, Miyakoshi N, Takahashi S, Tuoheti Y, Okada K, Shimada Y: Expression of growth factors in the early phase of supraspinatus tendon healing in rabbits. J Shoulder Elbow Surg 2006;15:371-377.

-55 Yamada M, Akeda K, Asanuma K, Thonar EJ, An HS, Uchida A, Masuda K: Effect of osteogenic protein-1 on the matrix metabolism of bovine tendon cells. J Orthop Res 2008;26:42-48.

-56 Zhang X, Ma Y, Fu X, Liu Q, Shao Z, Dai L, Pi Y, Hu X, Zhang J, Duan X, Chen W, Chen P, Zhou C, Ao Y: Runx2Modified Adipose-Derived Stem Cells Promote Tendon Graft Integration in Anterior Cruciate Ligament Reconstruction. Sci Rep 2016;6:19073.

-57 Li G, Yu F, Lei T, Gao H, Li P, Sun Y, Huang H, Mu Q: Bone marrow mesenchymal stem cell therapy in ischemic stroke: mechanisms of action and treatment optimization strategies. Neural Regen Res 2016;11:10151024.

-58 Lee JY, Zhou Z, Taub PJ, Ramcharan M, Li Y, Akinbiyi T, Maharam ER, Leong DJ, Laudier DM, Ruike T, Torina PJ, Zaidi M, Majeska RJ, Schaffler MB, Flatow EL, Sun HB: BMP-12 treatment of adult mesenchymal stem cells in vitro augments tendon-like tissue formation and defect repair in vivo. PLoS One 2011;6:e17531.

-59 Mazzocca AD, McCarthy MB, Chowaniec D, Cote MP, Judson CH, Apostolakos J, Solovyova O, Beitzel K, Arciero RA: Bone marrow-derived mesenchymal stem cells obtained during arthroscopic rotator cuff repair surgery show potential for tendon cell differentiation after treatment with insulin. Arthroscopy 2011;27:1459-1471.

60 Zhao L, Liu X, Zhang Y, Liang X, Ding Y, Xu Y, Fang Z, Zhang F: Enhanced cell survival and paracrine effects of mesenchymal stem cells overexpressing hepatocyte growth factor promote cardioprotection in myocardial infarction. Exp Cell Res 2016;344:30-39. 